\title{
Use of digital gene expression to discriminate gene expression differences in early generations of resynthesized Brassica napus and its diploid progenitors
}

\author{
Jinjin Jiang ${ }^{1}$, Yanlin Shao ${ }^{1}$, Kun Du', Liping Ran' ${ }^{1}$ Xiaoping Fang ${ }^{2}$ and Youping Wang ${ }^{1^{*}}$
}

\begin{abstract}
Background: Polyploidy is an important evolutionary mechanism in flowering plants that often induces immediate extensive changes in gene expression through genomic merging and doubling. Brassica napus $L$. is one of the most economically important polyploid oil crops and has been broadly studied as an example of polyploid crop. RNA-seq is a recently developed technique for transcriptome study, which could be in choice for profiling gene expression pattern in polyploids.
\end{abstract}

Results: We examined the global gene expression patterns of the first four generations of resynthesized $B$. napus $\left(\mathrm{F}_{1}-\mathrm{F}_{4}\right)$, its diploid progenitors B. rapa and B. oleracea, and natural B. napus using digital gene expression analysis. Almost 42 million clean tags were generated using Illumina technology to produce the expression data for 25959 genes, which account for $63 \%$ of the annotated B. rapa genome. More than 56\% of the genes were transcribed from both strands, which indicate the importance of RNA-mediated gene regulation in polyploidization. Tag mapping of the B. rapa genome generated 19023, 18547, 24383, 20659, 18881, 20692, and 19955 annotated genes for the $B$. rapa, $B$. oleracea, $F_{1}-F_{4}$ of synthesized $B$. napus, and natural $B$. napus libraries, respectively. The unambiguous tag-mapped genes in the libraries were functionally categorized via gene ontological analysis. Thousands of differentially expressed genes (DEGs) were identified and revealed the substantial changes in $F_{1}-F_{4}$. Among the 20 most DEGs are DNA binding/transcription factor, cyclin-dependent protein kinase, epoxycarotenoid dioxygenase, and glycine-rich protein. The Kyoto Encyclopedia of Genes and Genomes (KEGG) analysis of the DEGs suggested approximately 120 biological pathways.

Conclusions: The systematic deep sequencing analysis provided a comprehensive understanding of the transcriptome complexity of early generations of synthesized B. napus. This information broadens our understanding of the mechanisms of $B$. napus polyploidization and contributes to molecular and genetic research by enriching the Brassica database.

\section{Background}

Polyploidization is an ancient and ongoing evolutionary process that promotes plant evolution by reshaping genomes $[1,2]$. The majority of flowering plants have undergone polyploidization (complete or partial) and chromosome rearrangement [3]. Generally, polyploids are divided into allopolyploids and autopolyploids [4,5].

\footnotetext{
* Correspondence: wangyp@yzu.edu.cn

'Jiangsu Provincial Key Laboratory of Crop Genetics and Physiology,

Yangzhou University, Yangzhou 225009, China

Full list of author information is available at the end of the article
}

Many major crops, including wheat, cotton, oat, coffee, and oilseed rape, are fundamentally allopolyploids [6-8]. Recently, many studies have revealed that methylation status, gene expression profile, chromosomal rearrangements, deletions, insertions, and sequence substitutions in many allopolyploids differ to their progenitor $[9,10]$. Birchler and Veitia [11] proposed the gene balance theory with regard to quantitative traits and gene duplication following polyploidy [11]. Angiosperm genome plasticity in polyploids is always related to changes in gene expression, which are largely controlled by epigenetic profiles [12,13]. Gaeta and

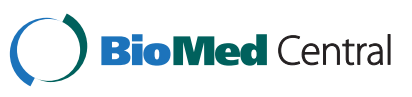


Pires [14] concluded that these genomic changes occur in both natural and resynthesized polyploids [14]. Molecular analyses of natural and resynthesized allopolyploids indicate that genetic and epigenetic changes are common results of polyploidization in different species $[5,15,16]$. Discovering the structural and functional evolution of genomes during polyploidization is of great importance in plant biology [17].

Cultivated Brassica species include important economical crops mostly closely related to Arabidopsis thaliana and provide great chances for studying the effects of polyploidization [3]. The availability of the Arabidopsis genome effectively facilitates studies on Brassica polyploidization [18]. Soon after the Arabidopsis and Brassica lineage diverged 17.0 million years ago (Mya), the triplicated Brassica subgenomes divergence was estimated to be 14.3 Mya $[17,19]$. B. rapa (A genome) and B. oleracea (C genome) descended from a common hexaploid ancestor of $A$. thaliana. Moreover, the lineage of B. rapa and B. oleracea diverged around 3.5 Mya [20], and a recent segmental duplication in B. rapa occurred $\sim 0.8$ Mya [21]. Studies on A- and C-genome mapping, genome comparison, and genome evolution have been performed during the past decades. Additional genome duplication aside from triplication, as well as complex chromosomal changes was revealed in B. oleracea [22,23]. B. rapa, $\sim 529 \mathrm{Mb}$ per haploid, was first launched for complete genome sequencing. The allopolyploid B. napus (AC, $\mathrm{n}=19)$ was spontaneously derived from hybridization of $\mathrm{A}$ and $\mathrm{C}$ progenitors [24]. The genome of natural B. napus was confirmed intact without rearrangement, but resynthesized $B$. napus underwent rapid changes in the early generations, including genetic changes and methylation changes [25-28]. Nonadditive proteins and additive proteins were completely identified in resynthesized $B$. napus, indicating the early steps of allopolyploidization repatterning are controlled by nonstochastic mechanisms [10,29]. Xiong et al. [30] indicated that aneuploidy, gross chromosomal rearrangements, and dosage balance maintain the genomic stability of synthesized B. napus [30].

Considering microarrays enable the comparison of gene expression at the transcriptome level, a set of Brassica unigenes assembled using Brassica expressed sequence tags (ESTs) was developed and assigned to discriminate paralogous genes, but not homologous genes, between the $\mathrm{A}$ - and the C-genome [6,31]. Recently emerged next generation sequencing (NGS) technology is an alternative method for better genome, epigenome, and transcriptome study [32,33]. Many plant species have benefited from this technology, including B. rapa and $B$. napus. The draft genome sequence of $B$. rapa accession Chiifu-401-42 was newly released by Illumina GA II technology and annotated [34], which provides an important resource for studying the evolution of polyploid genomes. Leaf transcriptome of $B$. napus had been dissected by sequencing $[35,36]$.

In the present research, we conducted a digital gene expression (DGE) analysis on resynthesized B. napus across the $\mathrm{F}_{1}-\mathrm{F}_{4}$ generations to address the transcriptome changes after polyploidization, which were also compared with their genetic progenitors ( $B$. rapa and $B$. oleracea) and natural $B$. napus. Previous studies on proteomic changes in this population revealed that differentially expressed proteins in $\mathrm{F}_{1}$ differed from the progenitors, exhibiting non-additive repatterning. Furthermore, gene silencing during polyploidization induces differences in protein expression in different generations of synthesized B. napus [37]. We report the expression profile of genes in resynthesized $B$. napus, show the upregulation of essential pathways and genes in $F_{1}$, and compare them with progenitors, which are downregulated in the $F_{2}-F_{4}$ generations. This is the first comprehensive transcriptomic research that identifies DEGs and the pathways involved in first four generations of synthesized B. napus after polyploidization.

\section{Results}

\section{Digital gene expression (DGE) profile}

To investigate global transcriptome during the polyploidization of $B$. napus, we performed a DGE analysis on the seedling stage of resynthesized $B$. napus $\left(\mathrm{F}_{1}-\mathrm{F}_{4}\right)$ and its diploid parents, as well as natural $B$. napus. Finally, DGE libraries from the leaves of four-week-old plants were generated and sequenced by Illumina technology. The sequence data are available from the GEO repository, accession number GSE43246. The statistics of the DGE tags is shown in Table 1. Approximately six million raw tags were generated for each library, and more than $97 \%$ of the raw tags were clean tags. A total of 6178564 raw tags were obtained from $B$. rapa $(\mathrm{Br}), 6059222$ from $B$. oleracea (Bo), 6155227 from B. napus $-\mathrm{F}_{1}\left(\mathrm{Bn}-\mathrm{F}_{1}\right), 6092805$ from B. napus $-\mathrm{F}_{2}\left(\mathrm{Bn}-\mathrm{F}_{2}\right), 6142098$ from B. napus $-\mathrm{F}_{3}$ $\left(\mathrm{Bn}-\mathrm{F}_{3}\right), 5938583$ from B. napus $-\mathrm{F}_{4}\left(\mathrm{Bn}-\mathrm{F}_{4}\right)$, and 5964594 from natural B. napus (Bn-N). After removing the lowquality sequences and adapter sequences, 6018254, 5930726, 6022170, 5950123, 5991210, 5798939, and 5823113 clean tags were obtained with 21 nt in length in the corresponding species. Unambiguous tags were counted and normalized to the number of transcripts per million tags (TPM) to evaluate the gene expression level. The results show that the mRNA transcribed from major genes had fewer than ten copies and only a small proportion of genes were highly expressed. The distribution of clean tags in the seven libraries was determined to evaluate the normality of the dataset. The results show a consistent pattern, with most of the tags coming from highly expressed genes. In this analysis, the total number of clean tags is the sum of all clean tags and the number of distinct 
Table 1 Statistics of categorization and abundance of DGE tags

\begin{tabular}{|c|c|c|c|c|c|c|c|c|}
\hline Summary & & B. rapa & B. oleracea & B. napus- $\mathrm{F}_{1}$ & B. napus- $\mathrm{F}_{2}$ & B. napus- $\mathrm{F}_{3}$ & B. napus- $F_{4}$ & B. napus- $\mathrm{N}$ \\
\hline Raw Data & Total & 6178564 & 6059222 & 6155227 & 6092805 & 6142098 & 5938583 & 5964594 \\
\hline Raw Data & Distinct Tag & 293575 & 243895 & 331406 & 275597 & 284202 & 272986 & 269285 \\
\hline Clean tag & Total number & 6018254 & 5930726 & 6022170 & 5950123 & 5991210 & 5798939 & 5823113 \\
\hline Clean tag & $\begin{array}{l}\text { Distinct Tag } \\
\text { number }\end{array}$ & 133499 & 116771 & 199428 & 134403 & 134721 & 134857 & 128967 \\
\hline Tag Mapping to Gene & Total number & 1964909 & 1747843 & 3025405 & 2354059 & 1652733 & 2295897 & 2253347 \\
\hline Tag Mapping to Gene & $\begin{array}{l}\text { Distinct Tag } \\
\text { number }\end{array}$ & 44267 & 36220 & 88270 & 49781 & 42613 & 51122 & 45358 \\
\hline $\begin{array}{l}\text { Unambiguous Tag } \\
\text { Mapping to Gene }\end{array}$ & Total number & 1679848 & 1475050 & 2495411 & 2016182 & 1415736 & 1971591 & 1924944 \\
\hline $\begin{array}{l}\text { Unambiguous Tag } \\
\text { Mapping to Gene }\end{array}$ & $\begin{array}{l}\text { Total \% of clean } \\
\text { tag }\end{array}$ & $27.91 \%$ & $24.87 \%$ & $41.44 \%$ & $33.88 \%$ & $23.63 \%$ & $34.00 \%$ & $33.06 \%$ \\
\hline $\begin{array}{l}\text { Unambiguous Tag } \\
\text { Mapping to Gene }\end{array}$ & $\begin{array}{l}\text { Distinct Tag } \\
\text { number }\end{array}$ & 39414 & 31933 & 79648 & 44456 & 37996 & 45762 & 40561 \\
\hline $\begin{array}{l}\text { Unambiguous Tag } \\
\text { Mapping to Gene }\end{array}$ & $\begin{array}{l}\text { Distinct Tag \% of } \\
\text { clean tag }\end{array}$ & $29.52 \%$ & $27.35 \%$ & $39.94 \%$ & $33.08 \%$ & $28.20 \%$ & $33.93 \%$ & $31.45 \%$ \\
\hline Tag-mapped Genes & number & 19023 & 18547 & 24383 & 20659 & 18881 & 20692 & 19955 \\
\hline Tag-mapped Genes & $\%$ of ref genes & $46.20 \%$ & $45.05 \%$ & $59.22 \%$ & $50.17 \%$ & $45.86 \%$ & $50.26 \%$ & $48.47 \%$ \\
\hline $\begin{array}{l}\text { Unambiguous } \\
\text { Tag-mapped Genes }\end{array}$ & number & 16574 & 15970 & 22059 & 18155 & 16479 & 18196 & 17448 \\
\hline $\begin{array}{l}\text { Unambiguous } \\
\text { Tag-mapped Genes }\end{array}$ & $\%$ of ref genes & $40.25 \%$ & $38.79 \%$ & $53.58 \%$ & $44.09 \%$ & $40.02 \%$ & $44.19 \%$ & $42.38 \%$ \\
\hline Mapping to Genome & Total number & 2437918 & 2105332 & 1776978 & 2290544 & 2658991 & 2232572 & 2164464 \\
\hline Mapping to Genome & $\begin{array}{l}\text { Total \% of clean } \\
\text { tag }\end{array}$ & $40.51 \%$ & $35.50 \%$ & $29.51 \%$ & $38.50 \%$ & $44.38 \%$ & $38.50 \%$ & $37.17 \%$ \\
\hline Mapping to Genome & $\begin{array}{l}\text { Distinct Tag } \\
\text { number }\end{array}$ & 44076 & 30703 & 49898 & 42334 & 45680 & 42590 & 40689 \\
\hline Mapping to Genome & $\begin{array}{l}\text { Distinct Tag \% of } \\
\text { clean tag }\end{array}$ & $33.02 \%$ & $26.29 \%$ & $25.02 \%$ & $31.50 \%$ & $33.91 \%$ & $31.58 \%$ & $31.55 \%$ \\
\hline Unknown Tag & Total number & 1615427 & 2077551 & 1219787 & 1305520 & 1679486 & 1270470 & 1405302 \\
\hline Unknown Tag & $\begin{array}{l}\text { Total \% of clean } \\
\text { tag }\end{array}$ & $26.84 \%$ & $35.03 \%$ & $20.25 \%$ & $21.94 \%$ & $28.03 \%$ & $21.91 \%$ & $24.13 \%$ \\
\hline Unknown Tag & $\begin{array}{l}\text { Distinct Tag } \\
\text { number }\end{array}$ & 45156 & 49848 & 61260 & 42288 & 46428 & 41145 & 42920 \\
\hline Unknown Tag & $\begin{array}{l}\text { Distinct Tag \% of } \\
\text { clean tag }\end{array}$ & $33.82 \%$ & $42.69 \%$ & $30.72 \%$ & $31.46 \%$ & $34.46 \%$ & $30.51 \%$ & $33.28 \%$ \\
\hline
\end{tabular}

Clean tags are tags after filtering dirty tags from raw data. Distinct tags are different kinds of tags and unambiguous tags are the reminder clean tags after removing tags mapped to reference genome.

clean tags is the number of different clean tags (Figure 1, Additional file 1: S1 and Additional file 2: S2). We found that the percentage of distinct tags with high counts dropped dramatically, and the distinct tags with more than 100 copies accounted less than $10 \%$. However, more than $70 \%$ of total clean tags have accounts above 100 in each library. By contrast, $50 \%$ of the distinct clean tags had copy numbers between two and five, but they only represented around $4 \%$ of the total number of clean tags, which indicates only a small number of mRNA were expressed at high abundance and the majority were expressed at very low levels [38]. The clean tags were then mapped onto the $B$ rapa genome [34] and the numbers of tags that could be mapped onto genes with a maximum of one base pair mismatch in $\mathrm{Br}$, Bo, Bn- $\mathrm{F}_{1}, \mathrm{Bn}-\mathrm{F}_{2}, \mathrm{Bn}-\mathrm{F}_{3}, \mathrm{Bn}-\mathrm{F}_{4}$, and $\mathrm{Bn}-\mathrm{N}$ were 1964909, 1747843, 3025405, 2354059, 1652733, 2295897, and 2253347, respectively (Table 1). Statistical analysis of clean tag alignment was conducted, including analysis of total clean tags and distinct clean tags (Additional file 3: S3, Additional file 2: S2). We found that around 70\% of total clean tags were mapped onto the $B$. rapa genome with perfect match or with $1 \mathrm{bp}$ mismatch to sense or anti-sense genes, and approximately $65 \%$ of the distinct clean tags were successfully mapped. Finally, the tag mapping onto the $B$. rapa genome generated 19023 tag-mapped genes for $\mathrm{Br}, 18547$ for Bo, 24383 for Bn-F, 20659 for $B n-F_{2}, 18881$ 


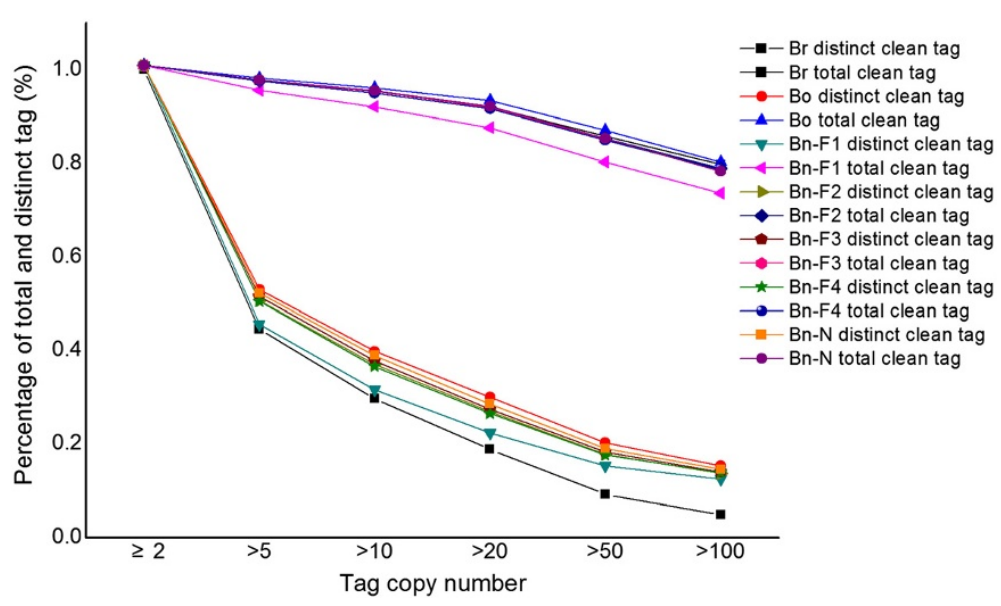

Figure 1 Distribution of total tag and distinct tag counts over different tag abundance categories from the seven libraries.

for $\mathrm{Bn}-\mathrm{F}_{3}, 20692$ for $\mathrm{Bn}-\mathrm{F}_{4}$, and 19955 for Bn-N. In total, 25959 genes were identified from the seven libraries, which accounted for approximately $63 \%$ of genes in the annotated B. rapa genome (Additional file 4: S4).

To determine whether the sequencing depth was sufficient for the transcriptome coverage, a saturation analysis was performed to check whether the number of detected genes increased with sequencing amount until the number of reads reached 2 million (Additional file 5: S5). We also analyzed the distribution of the ratio of distinct tag copy numbers in each pair of libraries and found that more than $90 \%$ of the distinct tags had ratios up to 5-fold (Additional file 6: S6). Antisense genes play an important role in gene expression and regulation, and about 36\% (14775) of the genes were matched by distinct tags in antisense orientations. Up to 5726 genes were mapped by DGE tags in both sense and antisense orientations in the Br library, 5123 in the Bo library, 11903 in the Bn-F library, $6568 \mathrm{Bn}-\mathrm{F}_{2}, 5692$ in the Bn- $\mathrm{F}_{3}$ library, 6887 in the Bn-F 4 library, and 6253 in the Bn-N library (Additional file 4: S4). In total, more than 56\% of the genes (14775 of 25959 genes) were transcribed from both strands, which indicate the importance of RNAmediated gene regulation in polyploidization.

\section{Differentially expressed genes in early generations of resynthesized $B$. napus}

To identify genes that were differentially expressed in the early generations of synthesized $B$. napus after polyploidization, we compared pairs of DGE profiles of the seven libraries $\left(\mathrm{Br}\right.$ versus $\mathrm{Bn}-\mathrm{F}_{1}$, Bo versus $\mathrm{Bn}-\mathrm{F}_{1}, \mathrm{Bn}-\mathrm{F}_{1}$ versus $B n-F_{2}, B n-F_{1}$ versus $B n-F_{3}, B n-F_{1}$ versus $B n-F_{4}$, and $\mathrm{Bn}-\mathrm{N}$ versus $\mathrm{Bn}-\mathrm{F}_{1}$, where $A$ was the control and $B$ was experimental group in 'A versus B') to analyze gene expression variations (Figure 2 and Additional file 7: S7). The comparison of $B$. napus $-\mathrm{F}_{1}$ with $B$. rapa revealed that 4197 DEGs were significantly upregulated and 360
DEGs were downregulated in B. napus $-\mathrm{F}_{1}$ in comparison with $B$. rapa. By contrast, 4554 DEGs were downregulated and 886 DEGs were upregulated in B. napus- $\mathrm{F}_{1}$ compared with $B$. oleracea. The number of upregulated DEGs in B. napus $-\mathrm{F}_{1}$ was more than that downregulated after polyploidization, which might indicate heterosis. Apart from the DEGs among resynthesized B. napus $-\mathrm{F}_{1}$ and its progenitors, the number of the identified DEGs differed in the early generations of synthesized B. napus, and most of the DEGs showed downregulation in the $\mathrm{F}_{2}-\mathrm{F}_{4}$ generations compared with the $\mathrm{F}_{1}$ generation. Up to 3022 DEGs were downregulated and 507 were upregulated in B. napus $-\mathrm{F}_{2}$ compared with $B$. napus $-\mathrm{F}_{1} ; 4718$ DEGs were downregulated and 502 were upregulated in B. napus $-\mathrm{F}_{3}$ compared with $B$. napus $-\mathrm{F}_{1} ; 2882$ DEGs were downregulated and 545 DEGs were upregulated in $B$. napus $-\mathrm{F}_{4}$ and $B$. napus $-\mathrm{F}_{1}$. Comparison of resynthesized and natural $B$. napus showed 649 tags were downregulated and 2916 tags were upregulated in B. napus $-\mathrm{F}_{1}$ in comparison with natural $B$. napus. By comparison, the lowest number of DEGs was found in the $\mathrm{F}_{4}$ generation, which was in a relatively stable stage after polyploidization. Figure 3 shows the distribution of genes commonly expressed in B. rapa, B. oleracea, B. napus $-\mathrm{F}_{1}$, and natural $B$. napus, which indicates that a number of conserved genes were identified apart from the DEGs. Distribution of genes commonly and specifically expressed in Bn- $\mathrm{F}_{1}$, $\mathrm{F}_{2}, \mathrm{~F}_{3}$ and $\mathrm{F}_{4}$ was also analyzed (Figure 4). Among the DEGs, the 20 most abundantly expressed genes that were upregulated or downregulated during polyploidization are listed in Additional file 8: S8. We found that the genes that encode DNA binding/transcription factor (Bra039065, Bra018796) were more prominent, which were downregulated in $\mathrm{F}_{1}$ compared with its progenitors and then upregulated in the $F_{2}-F_{3}$ generations compared with the $F_{1}$ generation. Genes encoding cyclin-dependent protein kinase (Bra016640), epoxycarotenoid dioxygenase (Bra020970), 


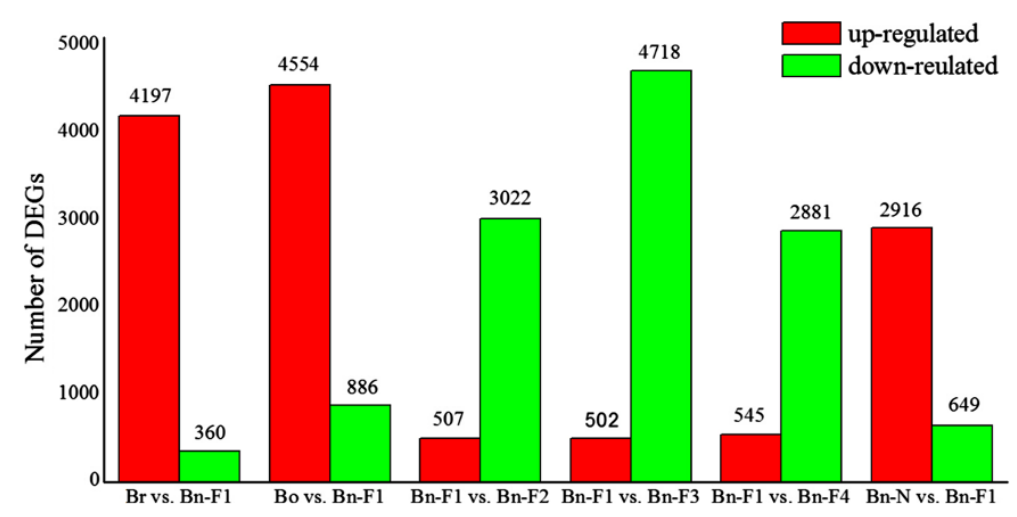

Figure 2 Numbers of differentially expressed genes in each comparison. The numbers of up-regulated (in red) and down-regulated genes (in green) are presented. 'A' was the control and 'B' was experimental group in 'A vs. B'.

and glycine-rich protein (Bra040817) were upregulated in $\mathrm{F}_{1}$ during polyploidization, and then downregulated in $\mathrm{F}_{2}-\mathrm{F}_{4}$ compared with $\mathrm{F}_{1}$.

\section{Functional annotation of DEGs}

Annotation of the sequences using GO database yielded good results for 22059 unambiguously mapped genes identified using the Bn- $\mathrm{F}_{1}$ DGE tags. These well-annotated sequences belonged to three main categories (cellular component, molecular function, and biological process) and distributed into 33 categories, including the most dominant pathways such as 'binding and catalytic activity', 'cell and cell part,' 'organelle,' 'cellular and metabolic processes', and 'response to stimulus' (Figure 5). A similar GO distribution was revealed in the other six libraries (Additional file 9: S9). However, we did not find any genes in the transporter cluster in Bo. Genes were not found in the extracellular region cluster in $\mathrm{Bn}-\mathrm{F}_{2}$. Enzyme regulators and translation regulators were not observed in $\mathrm{Bn}-\mathrm{F}_{3}$.

To identify the biological pathways active in the DGE libraries, we mapped all the annotated genes to terms in the KEGG database to search for significantly enriched genes involved in metabolic or signal transduction pathways (Additional file 10: S10). Among all the genes with KEGG annotation, DEGs were identified in $B n-F_{1}$ in comparison with $\mathrm{Br}$. In total, we assigned $2911 \mathrm{DEGs}$ to 121 KEGG pathways. Up to 38 of these pathways were significantly enriched, with $\mathrm{Q}$ values $\leq 0.05$ (red border region), including metabolic pathways, ribosome, and carbon fixation in photosynthetic organisms. Similar pathway enrichment was revealed in pair comparison of each libraries (Bo vs. $B n-F_{1}, B n-F_{1}$ vs. $B n-F_{2}, B n-F_{1}$ vs. $B n-F_{3}$, $\mathrm{Bn}-\mathrm{F}_{1}$ vs. $\mathrm{Bn}-\mathrm{F}_{4}$, and $\mathrm{Bn}-\mathrm{N}$ vs. $\mathrm{Bn}-\mathrm{F}_{1}$, where $\mathrm{A}$ was the control and $B$ was experimental group in 'A vs. B'). The 3339 DEGs identified in Bn- $\mathrm{F}_{1}$ in comparison with Bo were assigned to 122 KEGG pathways, 44 of which were significantly enriched. The 2293 DEGs identified in $\mathrm{Bn}-\mathrm{F}_{2}$ in comparison with $\mathrm{Bn}-\mathrm{F}_{1}$ were assigned to $122 \mathrm{KEGG}$ pathways, 3322 DEGs identified in $\mathrm{Bn}-\mathrm{F}_{3}$ in comparison with $\mathrm{Bn}-\mathrm{F}_{1}$ were assigned to 120 pathways, 2229 DEGs identified in $\mathrm{Bn}-\mathrm{F}_{4}$ in comparison with $\mathrm{Bn}-\mathrm{F}_{1}$ were assigned to 119 pathways, and 2283 DEGs identified in $\mathrm{Bn}-\mathrm{F}_{1}$ in comparison with $\mathrm{Bn}-\mathrm{N}$ were assigned to 119 pathways.

\section{Genome-wide non-additive gene regulation in synthesized $B n-F_{1}$}

We compared the transcript abundance in synthesized $\mathrm{Bn}-\mathrm{F}_{1}$ with the relative mid-parent value (MPV: an equal mixture of RNA from two parents) to identify genes that showed differential expression pattern in Bn- $F_{1}$. Up to 19785 genes in $\mathrm{Bn}-\mathrm{F}_{1}$ showed differences in expression from MPV, 12012 (60.7\%) of them showed higher expression levels than MPV, and 7773 (39.3\%) of these genes showed lower expressions than MPV. Among these nonadditively expressed genes, 9456 (47.8\%) genes were expressed at higher levels in $\mathrm{Br}$ than in Bo, and 10329

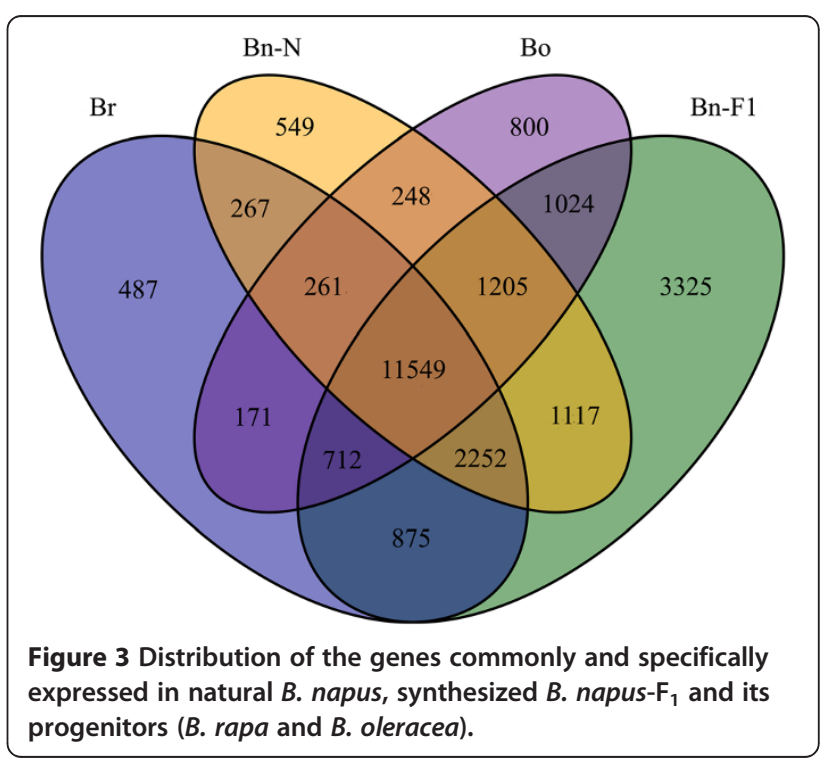




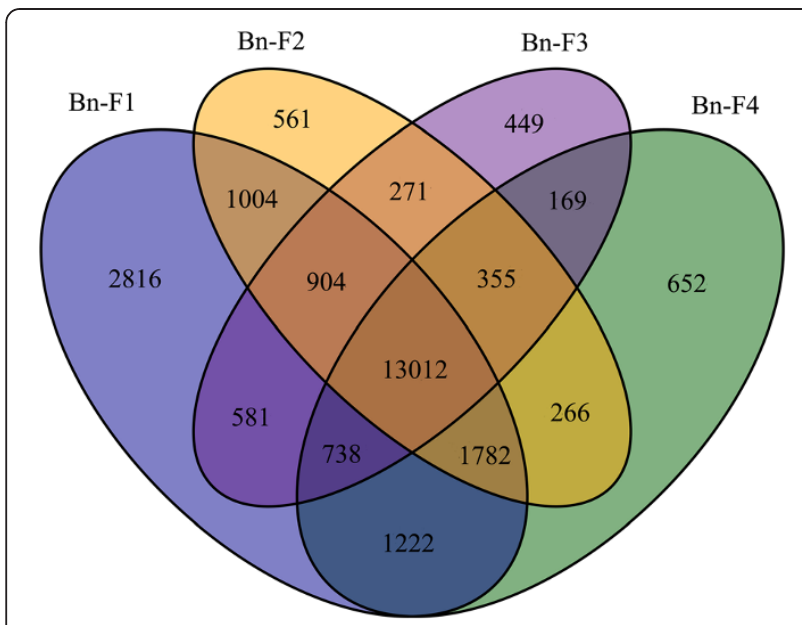

Figure 4 Distribution of the genes commonly and specifically expressed in synthesized $B$. napus $-F_{1}, B$. napus $-F_{2}, B$. napus $-F_{3}$ and $B$. napus $-\mathrm{F}_{4}$.

(52.2\%) genes were expressed at lower levels in $\mathrm{Br}$ than Bo (Table 2). The data suggest that the orthologous genes in polyploids are frequently expressed in a non-additive pattern.

\section{Cluster analysis of DEGs}

Cluster analysis of DEGs in the early generations of synthesized $B$. napus was performed with the correlated expression profile (Additional file 4: S4). Generally, 7948 DEGs in all the comparisons were clustered as the union of DEGs. Up to 1358 DEGs in all the comparisons were clustered as the DEG intersections (Figure 6). Among the 12 major clusters, the upregulated transcripts were enriched in clusters $\mathrm{A}-\mathrm{K}$ in the comparisons of $\mathrm{Bn}-\mathrm{N}$ vs. $\mathrm{Bn}-\mathrm{F}_{1}, \mathrm{Br}$ vs. $\mathrm{Bn}-\mathrm{F}_{1}$, and Bo vs. Bn- $\mathrm{F}_{1}$. The downregulated transcripts were enriched in clusters $A-K$ in the comparisons of $\mathrm{Bn}-\mathrm{F}_{1}$ vs. $\mathrm{Bn}-\mathrm{F}_{2}, \mathrm{Bn}-\mathrm{F}_{1}$ vs. $\mathrm{Bn}-\mathrm{F}_{3}$, and $\mathrm{Bn}-\mathrm{F}_{1}$ vs. Bn-F $\mathrm{F}_{4}$. Only one exception indicated that cluster $\mathrm{L}$ showed gene upregulation in $\mathrm{Bn}-\mathrm{F}_{2}, \mathrm{Bn}-\mathrm{F}_{3}$ and $\mathrm{Bn}-$ $\mathrm{F}_{4}$ compared with $\mathrm{Bn}-\mathrm{F}_{1}$, and downregulation in $\mathrm{Bn}-\mathrm{F}_{1}$ compared with $\mathrm{Bn}-\mathrm{N}, \mathrm{Br}$ and $\mathrm{Bo}$. We also identified the enrichment of DEGs in each cluster in different functional categories and found that the genes involved in the metabolism category were enriched significantly in all clusters except cluster $H$. Those in the protein with binding function or cofactor requirement category were enriched significantly in clusters $\mathrm{A}-\mathrm{H}$, whereas those in the subcellular localization category were enriched in all clusters except for cluster D. All the clustered DEGs are listed in Additional file 11: S11. Multiple genes represented for proteins with binding function, such as zinc ion binding (Bra019896), ATP binding (Bra008602, Bra023518), glutathione binding (Bra022815), selenium binding (Bra032756), and FAD/NADP/NADPH binding (Bra001931), showed different expression values in different generations of resynthesized B. napus and its progenitors. Some genes that encode ribosomal proteins were also changed during polyploidization.

\section{Discussion}

Differences in gene expression between synthesized

B. napus $-\mathrm{F}_{1}$ and its progenitors

Based on analysis of the leaf transcriptome data for synthesized $B$. napus $-\mathrm{F}_{1}$ and its progenitors, as well as the natural $B$. napus generated in this study, we found that the majority of the $B$. napus $-\mathrm{F}_{1}$ transcripts were conserved with the parental sequences. Up to 19023, 18547 , and 24383 sequences were mapped by $B$. napus$\mathrm{F}_{1}$ and its progenitors $B$. rapa and B. oleracea. 3182 genes with same TPM were expressed in $\mathrm{Bn}-\mathrm{F}_{1}$ and $\mathrm{Br}$, and 2421 genes with same TPM were expressed in Bn- $\mathrm{F}_{1}$

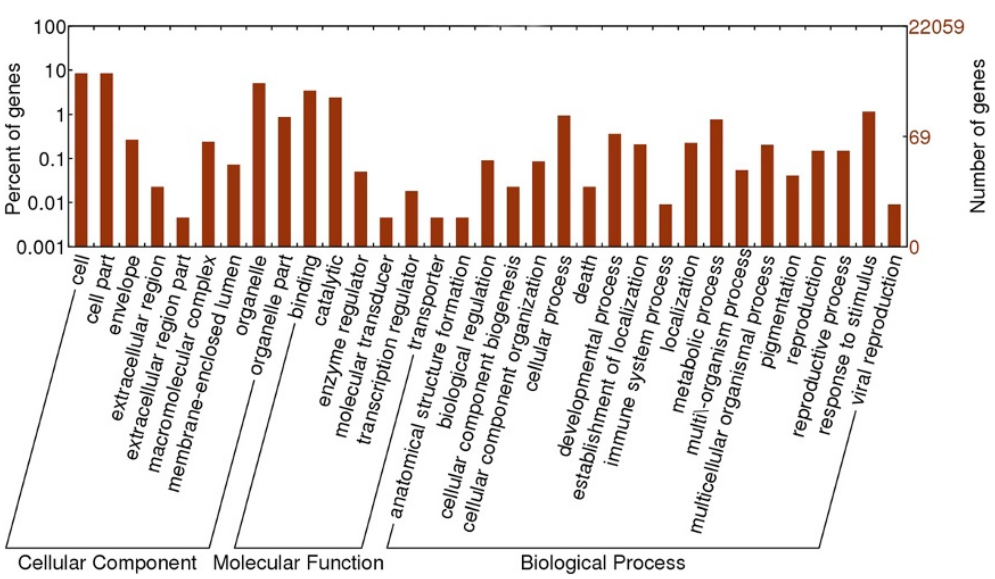

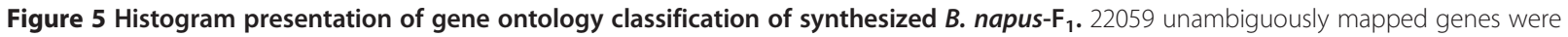
assigned to three main categories: cellular component, biological process and molecular function. The right- and left-axis indicates the number of genes in a category and the percentage of the specific category of genes in the main category, respectively. 
Table 2 Number of non-additively expressed genes in resynthesized Bn-F

\begin{tabular}{|c|c|c|c|c|c|c|c|c|}
\hline & a & $\%$ & b & $\%$ & b/a (\%) & c & $\%$ & c/a (\%) \\
\hline & \multicolumn{2}{|c|}{ No. of non-additively expressed genes } & \multicolumn{3}{|c|}{ No. of non-additively expressed genes } & \multicolumn{3}{|c|}{ No. of non-additively expressed genes } \\
\hline & \multicolumn{2}{|c|}{ Hybrid versus MPV } & \multicolumn{3}{|c|}{ Hybrid > MPV } & \multicolumn{3}{|c|}{ Hybrid < MPV } \\
\hline $\mathrm{Bn}-\mathrm{F}_{1}$ & 19785 & & 12012 & & 60.7 & 7773 & & 39.3 \\
\hline $\mathrm{Br}>\mathrm{Bo}$ & 9456 & 47.8 & 6134 & 51.1 & 64.9 & 3322 & 42.7 & 35.1 \\
\hline $\mathrm{Br}<\mathrm{BO}$ & 10329 & 52.2 & 5878 & 48.9 & 56.9 & 4451 & 57.3 & 43.1 \\
\hline
\end{tabular}

and Bo, which indicate that these genes might be significantly inherited by $\mathrm{Bn}-\mathrm{F}_{1}$ from one of its progenitors. The genes with different TPM expressed in B. napus $-\mathrm{F}_{1}$ and its progenitors, which indicate that the genome combination results duplicated genes in the resynthesized polyploid. However, only 14 of these commonly expressed genes showed TPM values in $\mathrm{Bn}-\mathrm{F}_{1}$ equal to the combination of its progenitors. Furthermore, most of the commonly expressed genes in the progenitors were non-additively expressed in $\mathrm{Bn}-\mathrm{F}_{1}$, which might be responsible for gene repression and activation [5,28,39]. The majority of the non-additively expressed genes in $\mathrm{Bn}-\mathrm{F}_{1}$ displayed expression values different to its progenitors, indicating that the transcriptome was reconciled during polyploidization [40]. Non-additive gene regulation should be involved in various biological pathways, which may lead to subfunctionalization of duplicated genes [41]. The gene expression in the resynthesized $\mathrm{Bn}-\mathrm{F}_{1}$ was more complicated than the simple combination of two genomes, which might be due to the homologous recombination between closely related genomes (A- and C-genome) [42]. Xiong et al. [30] reported the variable chromosome instability of synthesized $B$. napus, and chromosome loss was compensated by the gain of homologous chromosomes [30]. Szadkowski et al. [43] reported the genome blender of synthesized $B$. napus during first meiosis, which resulted in the rearrangement of two genomes and the restructuring in further generations [43]. Thus, resynthesized polyploids derived from closely related species result in changes in the non-additive pattern of multiple gene expression, which explains the molecular bases for hybrid vigor [44].

Besides the commonly expressed genes in polyploids and diploid progenitors, there are some species specific genes. As shown in Figure 3, 487, 800, 3325 and 549 genes were specifically expressed in $\mathrm{Br}, \mathrm{Bo}, \mathrm{Bn}-\mathrm{F}_{1}$ and $\mathrm{Bn}-\mathrm{N}$, respectively. The number of $B n-F_{1}$ specific genes was much higher than its progenitors, indicating many new transcripts emerged during polyploidization. This was consistent with $\mathrm{Xu}$ et al. [45] that 20 novel transcripts were detected in leaves of resynthesized Bn-F $\mathrm{F}_{1}$ [45]. Based on the clustering analysis of DEGs, we found 1333 out of the 1358 interspecific DEGs were up-regulated in Bn- $\mathrm{F}_{1}$ compared with $\mathrm{Br}$ and Bo. These up-regulated genes express proteins with different biological function, including salt tolerance protein, mitochondria/chloroplast membrance binding proteins, kinds of protein kinases, etc. Confirmation of these expression differences in further study is necessary for deep into the genetic causes of interest characteristics and improved qualities. Whereas 18 out of the 1358 interspecific DEGs were down-regulated in Bn- $\mathrm{F}_{1}$ compared with $\mathrm{Br}$ and Bo. Only two genes (Bra005226 and Bra026817) were up-regulated in $\mathrm{Bn}-\mathrm{F}_{1}$ compared with $\mathrm{Br}$, whereas down-regulated in $\mathrm{Bn}-\mathrm{F}_{1}$ compared with Bo. Five genes (Bra040268, Bra038457, Bra033201, Bra037320 and Bra039516) were up-regulated in Bn- $F_{1}$ compared with $\mathrm{Bo}$, whereas down-regulated in $\mathrm{Bn}-\mathrm{F}_{1}$ compared with $\mathrm{Br}$ (Figure 6). All these gene expression differences are of great interest for studying the genome polyploidization of Brassica species.

\section{Gene expression differences among early generations of} synthesized $B$. napus $\left(\mathrm{F}_{1}-\mathrm{F}_{4}\right)$ and natural $B$. napus

Hitherto, studies on polyploidy mechanism were conducted on natural and resynthesized $B$. napus using proteomic, transcriptome, and cytogenetic analyses [6,10,29,31,39,43]. However, few studies that trace genomic changes (including cytosine methylation, DNA fragment loss, genetic rearrangement) and transcriptome changes in different generations of synthesized $B$. napus have been reported $[9,27,28]$. As mentioned above, many novel transcripts emerged in $\mathrm{Bn}-\mathrm{F}_{1}$ compared its progenitors. The number of $\mathrm{Bn}-\mathrm{F}_{1}$ specific genes was also higher than $\mathrm{Bn}-\mathrm{F}_{1}, \mathrm{Bn}-\mathrm{F}_{2}$, $\mathrm{Bn}-\mathrm{F}_{3}$ (Figure 4). Many of these new transcripts emerged in the first generation of resynthesized B. napus disappeared after successive self hybridizations, indicating the instability of resynthesized polyploids. Based on the clustering of DEGs found in the comparisons of $\mathrm{Br} v \mathrm{vs}$. Bn- $\mathrm{F}_{1}$, Bo vs. $B n-F_{1}, B n-F_{1}$ vs. Bn- $F_{2}, B n-F_{1}$ vs. $B n-F_{3}, B n-F_{1}$ vs. $\mathrm{Bn}-\mathrm{F}_{4}$, and $\mathrm{Bn}-\mathrm{N}$ vs. $\mathrm{Bn}-\mathrm{F}_{1}$, we easily found that most DEGs are significantly downregulated in $\mathrm{Bn}-\mathrm{F}_{2}, \mathrm{Bn}-\mathrm{F}_{3}$, and $\mathrm{Bn}-\mathrm{F}_{4}$ compared with $\mathrm{Bn}-\mathrm{F}_{1}$, but these DEGs were upregulated in $\mathrm{Bn}-\mathrm{F}_{1}$ compared with its progenitors and natural $B$. napus. This phenomenon also indicated the genomic instability after polyploidization, and the discovery of multiple gene expression differences may compensate for the limitation of proteomic analysis [37]. The upregulation of DEGs in synthesized Bn- $\mathrm{F}_{1}$ during polyploidization could explain its improved characteristics compared with its progenitors. For example, the expression 


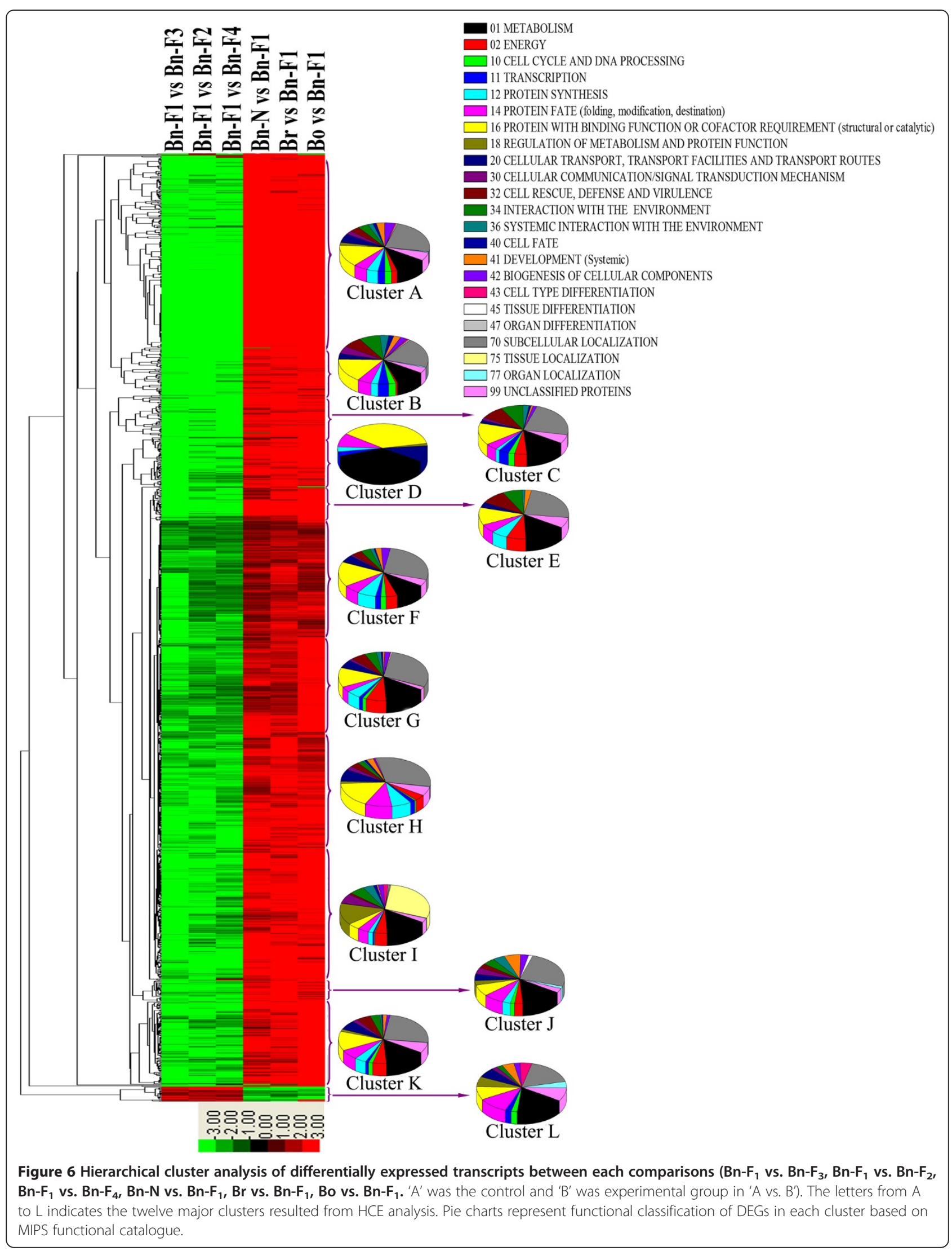


pattern of Bra022585, which encodes stress-responsive protein and Bra040633, which encodes defense-related protein, were changed during polyploidization. Some of these gene expression differences might be related to the DNA methylation or gene fragment losses during the combination of two genomes [45]. For example, Bra003009, which encodes O-methyltransferase 1, and Bra006323, which encodes an N2-dimethylguanosine tRNA methyltransferase family protein, were upregulated in $\mathrm{Bn}-\mathrm{F}_{1}$ during polyploidization and downregulated in the $\mathrm{F}_{2}-\mathrm{F}_{4}$ generations. In the pathway analysis, we found Bra003009 participated in the flavone and flavonol biosynthesis, which functioned in the transformation of kaempferide into kaempferol. This may indicate that kaempferol level was different in the synthesized $B$. napus. The changes in expression include many transcription factors, binding proteins, and ribosomal proteins, must be important for the merging of the A- and Cgenomes in synthesized polyploids. For the 18 DEGs, which were down-regulated in $\mathrm{Bn}-\mathrm{F}_{1}$ compared with $\mathrm{Br}$ and $\mathrm{Bo}$, we found they were up-regulated in $\mathrm{Bn}-\mathrm{F}_{2}, \mathrm{Bn}-\mathrm{F}_{3}$ and $\mathrm{Bn}-\mathrm{F}_{4}$ compared with $\mathrm{Bn}-\mathrm{F}_{1}$. This indicated that transcriptome recovery of genome balance functioned after polyploidization. Further research is needed to verify and determine the causes of these transcriptome changes and how they correlate with the phenotypic divergence and fertility of synthesized polyploids.

\section{Conclusions}

By applying the DGE deep sequencing, this study investigated the transcriptome profile of resynthesized $B$. napus and its diploid progenitors, as well as natural $B$. napus, with an aim to illustrate the gene expression differences during polyploidization. The amounts of transcripts obtained provided a foundation for future research on polyploidy mechanism of B. napus. Globally identified DEGs and putative biological pathways revealed the gene expression difference between synthesized B. napus and progenitors, and differential expression of genes across generations of synthesized $B$. napus. Generally, the gene expression in the resynthesized $B$. napus was more complicated than the simple combination of two genomes, and non-additive gene regulation was also detected in synthesized B. napus. These findings provided a contribution to the existing sequence resources for Brassica and would certainly facilitate polyploidy research of this genus.

\section{Methods}

\section{Plant material}

Seeds of resynthesized $B$. napus ( $\mathrm{F}_{1}$ generation) and its successive selfing generations $\left(\mathrm{F}_{2}-\mathrm{F}_{4}\right)$, its diploid progenitors B. rapa (cv. Aikangqing) and B. oleracea (cv. Zhonghua Jielan), were generously provided by the Oil Crops Research Institute of the Chinese Academy of Agricultural Sciences. Natural B. napus cv. Yang6 was provided by the Jiangsu Institute of Agricultural Science in the Lixiahe District (China). All the plants were cultivated in climate chambers at $25^{\circ} \mathrm{C}$, a 16 h light: $8 \mathrm{~h}$ dark photoperiod, and $70 \%$ relative humidity. The first true leaves from three plants of each genotype were pooled at the same physiologic stage (28-day-old seedlings) and frozen at $80^{\circ} \mathrm{C}$ for use.

\section{RNA preparation, Illumina RNA-sequencing and data processing}

Total RNA was extracted from leaves using RNAiso Plus (Takara) according to the manufacture's protocol. RNA concentrations were measured using a Qubit Fluorometer, and integrity was confirmed via 2100 Bioanalyzer (Agilent Technologies). The DGE libraries were prepared using Illumina Gene Expression Sample Prep Kit. Single-chain molecules were fixed onto a Solexa Sequencing Chip (flowcell) and sequenced by Illumina HiSeq ${ }^{\mathrm{Tm}} 2000$ System. Millions of raw, 35 bp sequences were generated. Image analysis, base calling, generation of raw tags, and the tags were counted using the Illumina pipeline [38]. Empty tags (no tag sequence between the adaptors), adaptors, low quality tags (tags containing one or more unknown nucleotides "N"), and tags with a copy number of 1 were removed from raw sequences to obtain clean tags (21 bp) containing CATG.

\section{Mapping of reads to the reference sequence}

To identify the gene expression patterns in early generations of synthesized $B$. napus, all clean tags were annotated by mapping to the sequenced genome of $B$. rapa [34] using the SOAP2 software, with a maximum of 1 nucleotide mismatch allowed [46]. All the tags mapped to reference sequences were filtered and the remaining tags were designated as ambiguous tags. Mapping events on both sense and antisense sequences were included in the data processing. For gene expression analysis, the number of expressed tags was calculated and then normalized to TPM (number of transcripts per million tags) $[47,48]$; and the DEGs were screened and used for mapping and annotation [49,50]. Gene annotation was conducted using Blast2GO [51]. The gene ontology (GO) categorization of all DEGs was displayed as three hierarchies for cellular component, molecular function, and biological process by searching in the GO database. Web Gene Ontology Annotation Plot (WEGO) was also used for GO classification of genes mapped by each DGE library [52]. Clustering analysis of differential gene expression pattern was also conducted using hierarchical clustering explorer (HCE) [53,54]. In this study, statistical analysis of DEGs among libraries was performed using stringent value FDR $\leq 0.001$ (false discovery rate) 
and the absolute value of $\mid \log 2$ Ratio $\mid \geq 1$ as the threshold of significant difference of gene expression. The DEGs in the hierarchical clustering were grouped into functional categories based on MIPS functional catalogue using Arabidopsis orthologues [55]. Pathway enrichment analysis of differential gene expression was conducted for further understanding gene function through blasting the KEGG database. A P-value of 0.05 was selected as the threshold for considering a gene set as significantly enriched.

\section{Additional files}

Additional file 1: S1. Distribution of total clean tags and distinct clean tags over different tag abundance categories in each DGE library. (A) Distribution of total tags. Numbers in the brackets of indicate the range of copy numbers for a specific category of tags. For example, [2,5] means all the tags in this category has 2 to 5 copies. Numbers in the parentheses show the total tag copy number for all the tags in that category. (B) Distribution of distinct tags. Numbers in the square brackets indicate the range of copy numbers for a specific category of tags. Numbers in the parentheses show the total types of tags in that category.

Additional file 2: S2. Summary of tag mapping in DGE analysis for seven libraries.

Additional file 3: S3. Mapping results of total tags and distinct tags of species in seven libraries. Normalized tag copy number was calculated by dividing tag counts for each gene with the total number of tags generated for each library and are presented per one million transcripts. PM and $1 \mathrm{MM}$ stand for perfect match and 1 miss match, respectively. (A) Mapping of total tags. (B) Mapping of distinct tags.

Additional file 4: S4. List of all genes identified by DGE. The first column represents names of the identified genes. Br_sense_raw and $\mathrm{Br}$ _antisense_raw mean the number of tags mapped to sense and antisense genes identified in DGE library of $\mathrm{B}$. rapa. Br_sense_norm and Br_antisense_norm mean total times of detected tags per million in DGE library of B. rapa. GO Component, GO Function and GO Process mean the three main categories (cellular component, molecular function and biological process) in the $\mathrm{GO}$ classification, respectively.

Additional file 5: S5. Sequencing saturation analysis of the seven libraries of $B$. rapa $(B r)$, B. oleracea (Bo), B. napus $-F_{1}\left(B n-F_{1}\right), B$. napus $-F_{2}$ $\left(B n-F_{2}\right), B$. napus $-F_{3}\left(B n-F_{3}\right), B$. napus- $F_{4}\left(B n-F_{4}\right)$ and natural B. napus $(\mathrm{Bn}-\mathrm{N})$. The number of detected genes was enhanced as the sequencing amount (total tag number) increased.

Additional file 6: S6. Distribution of ratio of distinct tag copy number in each pair of the libraries. 'A' was the control and ' $B$ ' was experimental group in 'A vs. B'.

Additional file 7: S7. List of differentially expressed genes in the early generations of resynthesized $B$. napus. DEGs between pairs of libraries were shown ( $B r$ vs. $B n-F_{1}, B$ vs. $B n-F_{1}, B n-F_{1}$ vs. $B n-F_{2}, B n-F_{1}$ vs. $B n-F_{3}, B n-$ $F_{1}$ vs. $B n-F_{4}, B n-N$ vs. $B n-F_{1}$. 'A' was the control and ' $B$ ' was experimental group in 'A vs. B'). TPM: transcript copies per million tags. Raw intensity: the total number of tags sequenced for each gene. FDR: false discovery rate. We used FDR $<0.001$ and the absolute value of log2Ratio $\leq 1$ as the threshold to judge the significance of gene expression difference. In order to calculate the log2Ratio and FDR, we used TPM value of 0.001 instead of 0 for genes that do not express in one sample.

Additional file 8: S8. The top 20 most up-regulated and downregulated genes between samples ( $B r$ vs. $B n-F_{1}, B$, vs. $B n-F_{1}, B n-F_{1}$ vs. $B n-$ $F_{2}, B n-F_{1}$ vs. $B n-F_{3}, B n-F_{1}$ vs. $B n-F_{4}, B n-N$ vs. Bn- $F_{1} .{ }^{\prime} A$ ' was the control and ' $B$ ' was experimental group in 'A vs. B'). TPM: transcript copies per million tags. This table does not include genes that only expressed in one sample.
Additional file 9: S9. Histogram presentation of gene ontology classification of B. rapa (Br), B. oleracea (Bo), B. napus $-F_{1}\left(B n-F_{1}\right), B$. napus- $F_{2}$ $\left(B n-F_{2}\right), B$. napus $-F_{3}\left(B n-F_{3}\right), B$. napus $-F_{4}\left(B n-F_{4}\right)$ and natural B. napus $(B n-N)$.

Additional file 10: S10. List of pathway enrichment analysis. Pathways with $Q$ value $\leq 0.05$ are significantly enriched in DEGs, see red-border region (' $A$ ' was the control and ' $B$ ' was experimental group in 'A vs. $B$ ').

Additional file 11: S11. Both union and intersection DEGs used for HCE clustering analysis.

\section{Competing interests}

The authors declare that they have no competing interests.

\section{Authors' contributions}

YW conceived and designed the study. JJ, YS and KD participated in the experiments. JJ, LR and XF analyzed the data. All authors drafted the manuscript and approved the final manuscript.

\section{Acknowledgments}

This work was supported by the NSFC project (31171581), Program of International S \&T Cooperation of China (1021), the Priority Academic Program Development of Jiangsu Higher Education Institutions, the project of Jiangsu Province (CXLX11_0998, CXZZ12_0897), and SRFDP (20123250110009).

\section{Author details}

${ }^{1}$ Jiangsu Provincial Key Laboratory of Crop Genetics and Physiology, Yangzhou University, Yangzhou 225009, China. ${ }^{2}$ Oil Crops Research Institute, Chinese Academy of Agricultural Sciences, Wuhan 430062, China.

Received: 2 October 2012 Accepted: 29 January 2013

Published: 1 February 2013

\section{References}

1. Wendel JF: Genome evolution in polyploids. Plant Mol Biol 2000, 42:225-249.

2. Soltis PS, Soltis DE: The role of hybridization in plant speciation. Annu Rev Plant Biol 2009, 60:561-588

3. Bancroft I: Duplicate and diverge: the evolution of plant genome microstructure. Trends Genet 2001, 17:89-93.

4. Jackson S, Chen ZJ: Genomic and expression plasticity of polyploidy. Curr Opin Plant Biol 2010, 13:153-159.

5. Chen ZJ: Genetic and epigenetic mechanisms for gene expression and phenotypic variation in plant polyploids. Annu Rev Plant Biol 2007, 58:377-406.

6. Higgins J, Magusin A, Trick M, Fraser F, Bancroft I: Use of mRNA-seq to discriminate contributions to the transcriptome from the constituent genome of the polyploidy crop species Brassica napus. BMC Genomics 2012, 13:247-260.

7. Pires JC, Zhao J, Schranz ME, Leon E, Quijada P, Lukens L, Osborn TC: Flowering time divergence and genomic rearrangements in resynthesized Brassica polyploids (Brassicaceae). Biol J Linn Soc 2004, 82:675-688.

8. Cifuentes M, Grandont L, Moore G, Chevre AM, Jenczewski E: Genetic regulation of meiosis in polyploid species: new insights into an old question. New Phytol 2010, 186:29-36.

9. Lukens LN, Pires JC, Leon E, Vogelzang R, Oslach L, Osborn T: Patterns of sequence loss and cytosine methylation within a population of newly synthesized Brassica napus allopolyploids. Plant Physiol 2006, 140:336-348.

10. Albertin W, Balliau T, Brabant P, Chevre AM, Eber F, Malosse C, Thiellement $H$ : Numerous and rapid nonstochastic modifications of gene products in newly synthesized Brassica napus allotetraploids. Genetics 2006, 173:1101-1113.

11. Birchler JA, Veitia RA: The gene balance hypothesis: implications for gene regulation, quantitative traits and evolution. New Phytol 2010, 186:54-62.

12. Adams KL, Wendel JF: Polyploidy and genome evolution in plants. Curr Opin Plant Biol 2005, 8:135-141.

13. Leitch $A R$, Leitch IJ: Genome plasticity and the diversity of polyploidy plants. Science 2008, 320:481-483

14. Gaeta RT, Pires JC: Homoeologous recombination in allopolyploids: the polyploidy ratchet. New Phytol 2010, 186:18-28. 
15. Doyle JJ, Flagel LE, Paterson AH, Rapp RA, Soltis DE, Soltis PS, Wendel JF: Evolutionary genetics of genome merger and doubling in plants. Annu Rev Genet 2008, 42:443-461.

16. Hegarty MJ, Hiscock SJ: Genomic clues to the evolutionary success of review polyploidy plants. Curr Biol 2008, 18:435-444

17. Cheung F, Trick M, Drou N, Lim YP, Park JY, Kwon SJ, Kim JA, Scott R, Pires JC, Paterson AH, Town C, Bancroft I: Comparative analysis between homoeologous genome segments of Brassica napus and its progenitor species reveals extensive sequence-level divergence. Plant Cell 2009, 21:1912-1928.

18. Paterson AH, Bowers JE, Burow MD, Draye X, Elsik CG, Jiang C, Katsar CS, Lan T, Lin Y, Ming R, Wright RJ: Comparative genomics of plant chromosomes. Plant Cell 2000, 12:1523-1539.

19. Lysak MA, Koch MA, Pecinka A, Schubert I: Chromosome triplication found across the tribe Brassiceae. Genome Res 2005, 15:516-525.

20. Inaba R, Nishio T: Phylogenetic analysis of Brassiceae based on the nucleotide sequences of amphidiploid Brassica napus (oilseed rape). Theor App/ Genet 2002, 105:1159-1165

21. Yang TJ, Kim JS, Kwon SJ, Lim KB, Choi BS, Kim JA, Jin M, Park JY, Lim MH, Kim HI, Lim YP, Kang JJ, Hong JH, Kim CB, Bhak J, Bancroft I, Park BS: Sequence-level analysis of the diploidization process in the triplicated FLOWERING LOCUS C region of Brassica rapa. Plant Cell 2006 18:1339-1347.

22. Lukens L, Zou F, Lydiate D, Parkin I, Osborn T: Comparison of a Brassica oleracea genetic map with the genome of Arabidopsis thaliana. Genetics 2003, 164:359-372

23. Wang XY, Torres MJ, Pierce G, Lemke C, Nelson LK, Yuksel B, Bowers JE, Marler B, Xiao YL, Lin LF, Epps E, Sarazen H, Rogers C, Karunakaran S, Ingles J, Gisttina E, Mun JH, Seol YJ, Park BS, Amasino RM, Quiros CF, Osborn TC, Pires JC, Town C, Paterson AH: A physical map of Brassica oleracea shows complexity of chromosomal changes following recursive paleopolyploidizations. BMC Genomics 2011, 12:470-484

24. $\cup$ N: Genome analysis in Brassica with special reference to the experimental formation of $B$. napus and its peculiar mode of fertilization. Japan J Genet 1935, 7:389-452.

25. Song KM, Lu P, Tang KL, Osborn TC: Rapid genome change in synthetic polyploids of Brassica and its implications for polyploidy evolution. Proc Natl Acad Sci USA 1995, 92:7719-7723.

26. Jenczewski E, Eber F, Grimaud A, Huet S, Lucas MO, Monod H, Chevre AM: PrBn, a major gene controlling homoeologous pairing in oilseed rape (Brassica napus) haploids. Genetics 2003, 164:645-653.

27. Gaeta RT, Pires JC, Iniguez-Luy F, Leon E, Osborn TC: Genomic changes in resynthesized Brassica napus and their effect on gene expression and phenotype. Plant Cell 2007, 19:3403-3417

28. Gaeta RT, Yoo SY, Piers JC, Doerge RW, Chen ZJ, Thomas C: Analysis of gene expression in resynthesized Brassica napus allopolyploids using Arabidopsis 70 mer oligo microarrays. PLoS One 2009, 4:4760-4773.

29. Albertin W, Alix K, Balliau T, Brabant P, Davanture M, Malosse C, Valot B, Thiellement H: Differential regulation of gene products in newly synthesized Brassica napus allotetraploids is not related to protein function nor subcellular localization. BMC Genomics 2007, 8:56-70.

30. Xiong ZY, Gaeta RT, Pires JC: Homoeologous shuffling and chromosome compensation maintain genome balance in resynthesized allopolyploid Brassica napus. Proc Natl Acad Sci USA 2011, 108:7908-7913.

31. Trick M, Cheung F, Drou N, Fraser F, Lobenhofer EK, Hurban P, Magusin A, Town CD, Bancroft I: A newly-developed community microarray resource for transcriptome profiling in Brassica species enables the confirmation of Brassica-specific expressed sequences. BMC Plant Biol 2009, 9:50-59.

32. Oshlack A, Robinson MD, Young MD: From RNA-seq reads to differential expression results. Genome Biol 2010, 11:220-229.

33. McIntyre LM, Lopiano K, Morse AM, Amin V, Oberg AL, Young LJ, Nuzhdin SV: RNA-seq: technical variability and sampling. BMC Genomics 2011, 12:293-305

34. Wang XW, Wang HZ, Wang J, Sun RF, Wu J, Liu SY, Bai YQ, Mun JH, Bancroft I, Cheng F, Huang SW, Li XX, Hua W, et al: The genome of mesopolyploid crop species Brassica rapa. Nature Genet 2011, 43:1035-1039.

35. Trick M, Long Y, Meng JL, Bancroft I: Single nucleotide polymorphysim (SNP) discovery in the polyploidy Brassica napus using Solexa transcriptome sequencing. Plant Biotechnol J 2009, 7:334-346.

36. Bancroft I, Morgan C, Fraser F, Higgins J, Wells R, Clissold L, Baker D, Long Y, Meng JL, Wang XW, Liu SY, Trick M: Dissecting the genome of the polyploidy crop oilseed rape by transcriptome sequencing. Nat Biotechnol 2011, 29:762-766.

37. Kong F, Mao SJ, Jiang JJ, Wang J, Fang XP, Wang YP: Proteomic changes in newly synthesized Brassica napus allotetraploids and their early generations. Plant Mol Biol Rep 2011, 29:927-935

38. Hoen PAC T, Ariyurek Y, Thygeson HH, Vreugdenhil E, Vossen RHAM Menezes RX, Boer JM, Ommen GJB, Dunnen JT: Deep sequencing-based expression analysis shows major advances in robustness, resolution and inter-lab portability over five microarray platforms. Nucl Acids Res 2008 36:141-151

39. Marmagne A, Brabant $P$, Thiellement $H$, Alix $K$ : Analysis of gene expression in resynthesized Brassica napus allotetraploids: transcriptional changes do not explain differential protein regulation. New Phytol 2010, 186:216-227.

40. Wang JL, Tian L, Lee HS, Wei NE, Jiang HM, Watson B, Madlung A, Osborn TC, Doerge RW, Comai L, Chen ZJ: Genome wide nonadditive gene regulation in Arabidopsis allotetraploids. Genetics 2006, 172:507-517.

41. Adams KLR, Gronn R, Percifield R, Wendel JF: Genes duplicated by polyploidy show unequal contributions to the transcriptome and organspecific reciprocal silencing. Proc Natl Acad Sci USA 2003, 100:4649-4654.

42. Mason AS, Huteau V, Eber F, Coriton O, Yan GJ, Nelson MN, Cowling WA Chevre AM: Genome structure affects the rate of autosyndesis and allosyndesis in $A A B C, B B A C$ and CCAB Brassica interspecific hybrids. Chromosome Res 2010, 18:655-666.

43. Szadkowski E, Huteau V, Lode M, Huneau C, Belcram H, Coriton O, Manzanares-Dauleux MJ, Delourme R, King GJ, Chalhoub B, Jenczewski E, Chevre AM: The first meiosis of resynthesized Brassica napus, a genome blender. New Phytol 2010, 186:102-112.

44. Birchler JA, Auger DL, Riddle NC: In search of the molecular basis for heterosis. Plant Cell 2003, 15:2236-2239.

45. Xu YH, Zhong L, Wu XM, Fang XP, Wang JB: Rapid alterations of gene expression and cytosine methylation in newly synthesized Brassica napus allopolyploids. Planta 2009, 229:471-483.

46. Li RQ, Yu C, Li YR, Lam TW, Yiu SM, Kristiansen K, Wang J: SOAP2: an improved ultrafast tool for short read alignment. Bioinformatics 2009, 25:1966-1967

47. Hoen PAC, Ariyurek $Y$, Thygesen HH, Vreugdenhil E, Vossen RHAM, Menezes RX, Boer JM, Ommen GJB, Dunnen JT: Deep sequencing-based expression analysis shows major advances in robustness, resolution and inter-lab portability over five microarray platforms. Nucl Acids Res 2008, 36:141-151.

48. Morrissy AS, Morin RD, Delaney A, Zeng T, McDonald H, Jones S, Zhao YJ, Hirst M, Marra MA: Next-generation tag sequencing for cancer gene expression profiling. Genome Res 2009, 19:1825-1835.

49. Audic S, Claverie JM: The significance of digital gene expression profiles. Genome Res 1997, 7:986-995

50. Benjamini BY, Yekutieli D: The control of the false discovery rate in multiple testing under dependency. Ann Stat 2011, 29:1165-1188.

51. Conesa A, Gotz S, Garcia-Gomez JM, Terol J, Talon M, Robles M: Blast2GO: a universal tool for annotation, visualization and analysis in functional genomics research. Bioinformatics 2005, 21:3674-3676.

52. Ye J, Fang L, Zheng H, Zhang Y, Chen J, Zhang Z, Wang J, Li S, Li R, Bolund L, Wang J: WEGO: a web tool for plotting GO annotations. Nucl Acids Res 2006, 34:293-297.

53. Eisen MB, Spellman PT, Brown PO, Botstein D: Cluster analysis and display of genome-wide expression. Proc Natl Acad Sci USA 1998, 95:14863-14868.

54. Saldanha AJ: Java treeview-extensible visualization of microarray data. Bioinformatics 2004, 20:3246-3248.

55. Ruepp A, Zollner A, Maier D, Albermann K, Hani J, Mokrejs M, Tetko I, Guldener U, Mannhaupt G, Munsterkotter M, Mewes HW: The FunCat, a functional annotation scheme for systematic classification of proteins from whole genomes. Nucl Acids Res 2004, 32:5539-5545.

doi:10.1186/1471-2164-14-72

Cite this article as: Jiang et al.: Use of digital gene expression to discriminate gene expression differences in early generations of resynthesized Brassica napus and its diploid progenitors. BMC Genomics 2013 14:72 